\title{
SOME RESULTS ON TEST ELEMENTS
}

\author{
by IAN M. ABERBACH* and BRIAN MACCRIMMON
}

(Received 24th September 1997)

\begin{abstract}
Let $(R, m)$ be a Noetherian local reduced ring of positive prime characteristic. We show that if $R$ is $Q$ Gorenstein then the test ideal of $R$ localizes, which extends a result of K. E. Smith. We also show that if $R_{c}$ is weakly F-regular and $Q$-Gorenstein, then $c$ has a power which is a completely stable test element. This extends results of Hochster and Huneke.
\end{abstract}

1991 Mathematics subject classification: 13A35.

\section{Introduction}

Throughout this paper all rings are commutative Noetherian of prime characteristic.

Perhaps the most central notion in tight closure theory is that of test elements. The existence of uniform annihilation plays a key role in several important commutative algebra theorems and so understanding test elements, which uniformly annihilate tight closure, is of fundamental importance. Questions about test elements can be divided into two types, localization and existence, but ultimately these are facets of each other. At present, the best theorems that are available exist under a Gorenstein hypothesis. The goal of this paper is to demonstrate that the Gorenstein condition in these theorems can be weakened; in particular we show that it is sufficient that some symbolic power of the canonical module be one-generated.

What makes the theory difficult is the localization problem for tight closure: it is unknown whether $I^{*} R_{\mathrm{P}}=\left(I R_{P}\right)^{*}$. Nevertheless, if the ideal $I$ is generated by a regular sequence this statement is true. The reason that matters are so well understood in the Gorenstein case is that to prove theorems for all ideals it is frequently sufficient to prove them for irreducible ideals primary to a maximal ideal and thus, in Gorenstein rings, for ideals generated by a regular sequence. In an arbitrary Cohen-Macaulay ring these irreducible ideals can get quite complicated. If $R$ is a normal Cohen-Macaulay domain with canonical ideal $J$, then the typical irreducible ideal is $\left(J, x_{2}, \ldots, x_{n}\right)$ with $x_{2}, \ldots, x_{n}$ parameters on $R / J$. Of course, if $R$ is Gorenstein then we may take $J=x_{1}$ and the theory is well developed, but we may also look at $R$ when there exists an $h$ such that $J^{(h)}=x_{1} R$. We will use the term $\mathbb{Q}$-Gorenstein for such rings, omitting the usual requirement that $R$ be normal. Even though we do not know whether localization commutes with tight

* The first author was partially supported by the NSF and by the University of Missouri Research Board. 
closure for irreducible ideals in $\mathbb{Q}$-Gorenstein rings, it seems to be almost true and it is close enough to give improved versions of the theorems on test elements.

For a reduced ring $R$, a test element is an element $c$ not in any minimal prime of $R$ such that $c I^{*} \subseteq I$ for all ideals $I$ of the ring. The test ideal, $\tau$, is the ideal generated by all test elements. Smith shows that if $R$ is a complete local Gorenstein ring then $\tau R_{P}$ is the test ideal for $R_{P}$ [7] (more generally she proves that parameter test ideals localize in complete Cohen-Macaulay rings [8]). In Section 3 we show that test ideals localize for Cohen-Macaulay $\mathbb{Q}$-Gorenstein reduced local rings. This gives several results on the openness of loci and existence of test elements, but the local condition is restrictive and so in Section 4 we use the gamma construction to get around this.

It is of great importance to have elements which not only annihilate tight closure over the ring but also over completions and localizations. We call a nonzero $c \in R$ a completely stable test element if it is a test element in every local ring of $R$ and also in the completion of every local ring. The strongest result on the existence of (completely stable) test elements is the following [4, Theorem 6.2]: Let $R$ be a reduced algebra of finite type over a reduced excellent local ring. Let $c$, not in any minimal prime, be such that $R_{c}$ is F-regular and Gorenstein. Then $c$ has a power which is a completely stable test element. In fact, in Section 4 we show that the hypothesis can be softened to $R_{c}$ being $\mathbb{Q}$-Gorenstein.

If all ideals of a ring are equal to their tight closure (i.e., the ring is weakly Fregular) then the test ideal is the unit ideal. It is conjectured that the completely stable test ideal is also the unit ideal. Using that any two dimensional F-rational ring is $\mathbb{Q}$ Gorenstein, we also show in Section 4 that the completely stable test ideal in a local excellent weakly F-regular ring has height at least three.

\section{Background}

Tight closure. We need the following notation and information from [3].

Let $F^{e}: R \rightarrow R$ denote the $e$-th iteration of the Frobenius endomorphism, so for $r \in R, F^{e}(r)=r^{q}$ where $q=p^{e}$.

Let $S$ denote $R$ considered as an $R$-algebra via the $e$-th power of the Frobenius endomorphism. The $e$-th Peskine-Szpiro functor, $F^{e}(-)=S \otimes_{R}(-)$, is a covariant functor from $R$-modules to $S$-modules, that is from $R$-modules to $R$-modules. Note that $a(b \otimes m)=(a b) \otimes m$ and $b \otimes a m=b a^{q} \otimes m$.

There is a natural map $M \rightarrow F^{e}(M)$ given by $m \mapsto 1 \otimes m$. We denote the image of $m$ in $F^{e}(M)$ by $m^{q}$. Let $N \subseteq M$ be $R$-modules. By $N_{M}^{[q]}$, or simply $N^{[q]}$, we mean the submodule of $F^{e}(M)$ generated by the image of $F^{e}(N)$, i.e., $\operatorname{Im}\left(F^{e}(N) \rightarrow F^{e}(M)\right.$ ). Thus $N^{[q]}$ is the submodule of $F^{e}(M)$ generated by $\left\{n^{q}: n \in N\right\}$. Also for $\eta \in N$ we use $\eta^{[q]}$ to denote the image of $\eta$ in $N^{[q]}$. In the case $M=R$ and $N=I$, an ideal, we have $F^{e}(R)=R$ and $I^{[q]} \subseteq R$ is the ideal generated by $\left\{i^{q}: i \in I\right\}$.

We can now define tight closure. Denote the complement of the minimal primes of $R$ by $R^{\circ}$. For $R$-modules $N \subseteq M$, we say that $x \in M$ is in the tight closure of $N$ in $M$, denoted $N_{M}^{*}$ (or simply $N^{*}$ if $M$ is understood), if there exists $c \in R^{\circ}$ such that $c x^{q} \in N_{M}^{[q]}$ for all $q \gg 0 . N$ is said to be tightly closed in $M$ if $N^{*}=N$. Tight closure is truly a closure operation if $M$ is a finitely generated module. 
Another notion of tight closure arises for non-Noetherian modules. The finitistic tight closure of a finitely generated module $N$ in $M$ is the union of the submodules $N_{M^{*}}^{*}$ where $M^{\prime}$ is any finitely generated submodule of $M$ containing $N$. This is denoted $N_{M}^{* f g}$ and $N_{M}^{* f g} \subseteq N_{M}^{*}$.

A ring $R$ is called weakly $F$-regular if every ideal in $R$ is tightly closed. The ring $R$ is then normal and under mild conditions (e.g., $R$ is the image of a Cohen-Macaulay ring) $R$ is Cohen-Macaulay. The term $F$-regular is reserved for rings for which all localizations are weakly F-regular. A ring is called $F$-rational if all ideals generated by parameters are tightly closed.

For reduced rings, $R$, we call $R F$-finite if $R^{1 / p}$ is module-finite over $R$. An F-finite ring is said to be strongly $F$-regular if for all $c$ not in any minimal prime of $R$, there exists $q$ such that the inclusion $R c^{1 / q} \subseteq R^{1 / q}$ splits as a map of $R$-modules.

For a reduced ring $R$, a test element is an element $c$ not in any minimal prime of $R$ such that $c I^{*} \subseteq I$ for all ideals $I$ of the ring, whereas the test ideal, $\tau$, is the ideal generated by all such elements. Then for the local ring $(R, m),[3,8.23]$ states that $\tau=\operatorname{Ann}_{R} 0_{E(R / m)}^{* f g}=\cap_{t}\left(I_{t}: I_{t}^{*}\right)$ for any sequence of Gorenstein ideals, $\left\{I_{t}\right\}$, cofinal with the powers of $m$. A completely stable test element is a test element which remains a test element in the completion of every local ring (this implies that it is also a test element in every local ring as well $[3,8.13(\mathrm{c})])$.

$\mathbb{Q}$-Gorenstein rings. Let $R$ be any ring and let $I \subseteq R$ be a height 1 ideal. By $I^{(h)}$ we mean the intersection of all height one primary components of $I^{h} . R$ is called $\mathbb{Q}$ Gorenstein if there exists a height one ideal $J$ that is a canonical module and such that $J^{(h)}$ is principal for some integer $h$. Here we do not assume $R$ is either normal or Cohen-Macaulay, although if $R$ is a Cohen-Macaulay reduced local ring which admits a canonical module (i.e., the homomorphic image of a Gorenstein local ring) then it has a canonical ideal of pure height one. $\mathbb{Q}$-Gorenstein weakly F-regular F-finite rings are strongly F-regular [6].

Q-Gorenstein implies Gorenstein at height one primes which are Cohen-Macaulay. One can see this by looking at the reduction of the canonical ideal, $J$, in a onedimensional Cohen-Macaulay $\mathbb{Q}$-Gorenstein ring, $R$. There exists $m$ such that $J^{m+1}=$ $b J^{m}$. Also since there are no embedded components $J^{h}=J^{(h)}=(a)$. Pick $k$ so that $k h>m$. Then $J^{k h+1}=b J^{k h}$ and hence by cancelling $J=(b)$.

Remark 2.1. If $R$ is Gorenstein at height 1 primes, then there are elements $a \in J$ and $b \in R$ such that $J=(a: b)$. To see this, let $U$ be the complement of the associated primes of $J$. Then $J U^{-1} R$ is principal and we may pick a generator $a \in J$. Then there is an element $b \in U$ such that $b J \subseteq(a)$. In fact, $J=(a: b)$, which can be checked at minimal primes of $a R$. Note that then for all $n, b^{n} J^{(n)} \subseteq\left(a^{n}\right)$.

\section{Localization of test ideals}

Smith in [8] proves that parameter test ideals localize for complete local CohenMacaulay reduced rings. One of the main points is that there is a natural action of 
Frobenius on $H_{m}^{d}(R)$, i.e., for a submodule $M$ of $H_{m}^{d}(R)$ there is a containment $F(M) \subseteq H_{m}^{d}(R)$. This is quite useful for the study of the parameter test ideal, which can be expressed as $\operatorname{Ann}_{R}\left(0_{H^{d}(R)}^{*}\right)$. Since the test ideal is $\operatorname{Ann}_{R}\left(0_{E}^{* f g}\right)$ one would like a comparable situation for the Frobenius action on the injective hull $E$ of the residue field; however there are no longer isomorphisms between $E$ and $F^{e}(E)$ and this makes an analogous definition of "F-ideals" rather problematic. Even worse, the maps in the limit system defining $F^{e}(E)$ are no longer injective. Nonetheless in the case when the ring is $\mathbb{Q}$-Gorenstein this bad behaviour can be controlled.

Previously, using methods similar to those in [8], Smith had proved that test ideals localize for complete local Gorenstein reduced rings [7, 4.1] (note that for Gorenstein rings the test ideal is the same as the parameter test ideal). It is this earlier result we extend here to: test ideals localize for local Cohen-Macaulay $\mathbb{Q}$-Gorenstein reduced rings. Notice that the condition of complete can be removed and in fact it can also be removed from [7, 4.1] by a direct application of Lemma 4.2 (rather than using the colocalization technique $[7,3.1])$. Unfortunately, since it is unknown whether test ideals behave well with respect to localization at maximal ideals, we are unable to remove the local condition. This is particularly desirable since it would give the existence of test elements without using the gamma construction (cf. 4.2).

If $J$ is a canonical ideal for the local Cohen-Macaulay ring $(R, m)$, choose $x_{1} \in J$ and $x_{2}, \ldots, x_{n} \in m$ such that $x_{1}, \ldots, x_{n}$ is a system of parameters and $x_{2}, \ldots, x_{n}$ is a system of parameters on $R / J$. Then the injective hull of the residue field over $R$ is $E=\lim _{\rightarrow s} H_{m}^{n-1}\left(R / x_{1}^{s} J\right)=\lim _{\longrightarrow} R /\left(x_{1}^{s} J, x_{2}^{s}, \ldots, x_{n}^{s}\right)$ (the maps are multiplication by $\left.x_{1} \cdots x_{n}\right)$. We can represent an element $\eta \in E$ by $\eta=\left[z+\left(x_{1}^{s} J, x_{2}^{s}, \ldots, x_{n}^{s}\right)\right]$.

Furthermore, if $J$ is principal at height one primes then $F^{e}(E) \cong F^{e}\left(\lim _{\longrightarrow} H_{m}^{n-1}\left(R / x_{1}^{s} J\right)\right) \cong$ $\lim _{\longrightarrow} H_{m}^{n-1}\left(R / x_{1}^{s q} J^{[q]}\right) \cong \lim _{\longrightarrow} H_{m}^{n-1}\left(R / x_{1}^{s q} J^{(q)}\right)$ (the last isomorphism follows because $\operatorname{dim}\left(J^{(q)} /\right.$ $\left.\left.\overrightarrow{J^{[q]}}\right) \leq n-2\right)$. Then $\eta^{[q]} \in F^{e}(E)$ may be identified with $\left[z^{q}+\left(x_{1}^{q s} J^{(q)}, x_{2}^{q s}, \ldots, x_{n}^{q s}\right)\right]$ (or with $\left.\left[z^{q}+\left(x_{1}^{q s} J^{[q]}, x_{2}^{q s}, \ldots, x_{n}^{q s}\right)\right]\right)$.

The first difficulty is that since the injective hull is never Noetherian when $\operatorname{dim} R>0$, we must ostensibly distinguish between the tight closure and the finitistic tight closure. The following proposition shows we need not distinguish between the finitistic tight closure and standard tight closure of zero in the injective hull over $\mathbb{Q}$ Gorenstein rings. For integers $n$ and $m$ we use $\lfloor n / m\rfloor$ to denote the floor of $n / m$, i.e., the greatest integer less than or equal to $n / m$.

Proposition 3.1. Let $R$ be a local Cohen-Macaulay $\mathbb{Q}$-Gorenstein ring. Then $0_{E}^{* g}=0_{E}^{*}$.

Proof. Let $J \subseteq R$ be a canonical ideal. Suppose that $J^{(h)}=\left(x_{1}\right)$, and $x_{2}, \ldots, x_{n}$ are parameters on $R / J$. We may assume that $x_{2} J \subseteq(a)$ for $a \in J$ as in Remark 2.1. Identify $E$ with $\lim R /\left(x_{1}^{s} J, x_{2}^{s}, \ldots, x_{n}^{s}\right)$ as above.

Take $\vec{\eta}=\left[z+\left(x_{1}^{s} J, x_{2}^{s} \ldots, x_{n}^{s}\right)\right] \in 0_{E}^{*}$. Then for some $c \in R^{\circ}, c \eta^{|q|}=\left[c z^{q}+\left(x_{1}^{s q} J^{|q|}\right.\right.$, $\left.\left.x_{2}^{s q}, \ldots, x_{n}^{s q}\right)\right]=0$ for all $q$. Translating this statement back to the ring, we get for each $q$ there is a $t_{q} \in \mathbb{N}$ such that $c\left(x_{1} \cdots x_{n}\right)^{t_{q}} z^{q} \in\left(x_{1}^{s q+t_{q}} J^{[q]}, \ldots, x_{n}^{s q+t_{q}}\right)$. But then $c\left(x_{1} \cdots x_{n}\right)^{t_{q}} z^{q} \in\left(x_{1}^{s q+t_{q}} x_{1}^{l_{t}^{q} 1}, \ldots, x_{n}^{s q+t_{q}}\right)$, and thus $c z^{q} \in\left(x_{1}^{s q} x^{\left.l_{h}^{q}\right)}, \ldots, x_{n}^{s q}\right)$. Hence $x_{1}^{h} c z^{q} \in$ 
$\left(x_{1}^{s q} J^{(q)}, \ldots, x_{n}^{s q}\right)$, which in turn gives $x_{1}^{h} c\left(x_{1} \cdots x_{n}\right)^{q} z^{q} \in\left(x_{1}^{(s+1) q} a^{q}, x_{2}^{(s+1) q}, \ldots, x_{n}^{(s+1) q}\right)$. Thus $\left(x_{1} \cdots x_{n}\right) z \in\left(x_{1}^{s+1} J, x_{2}^{s+1}, \ldots, x_{n}^{s+1}\right)^{*}$, which shows that $\eta \in 0_{E}^{* f g}$.

Lemma 3.2. Let $R$ be a local reduced Cohen-Macaulay $\mathbb{Q}$-Gorenstein ring. Let $\tau$ be the test ideal for $R$ and for a prime ideal, $P$, let $\tau_{P}$ be the test ideal for $R_{P}$. Then $\mathrm{Ann}_{E(R / P)} \tau R_{P} \subseteq 0_{E(R / P)}^{*}$ implies $\tau_{P} \subseteq \tau R_{P}$.

Proof. First observe that $A \operatorname{Ann}_{R_{P}}\left(\mathrm{Ann}_{E(R / P)} \tau R_{P}\right)=\tau R_{P}$, as this is true for any ideal. If $\mathrm{Ann}_{E(R / P)} \tau R_{P} \subseteq 0_{E(R / P)}^{*}=0_{E(R / P)}^{* f g}$ then $\tau_{P}=$ Ann $0_{E(R / P)}^{*} \subseteq \mathrm{Ann}_{R_{P}}\left(\mathrm{Ann}_{E(R / P)} \tau R_{P}\right)=\tau R_{P}$

The next result generalizes $[7,4.1]$.

Theorem 3.3. Let $R$ be a local reduced Cohen-Macaulay $\mathbb{Q}$-Gorenstein ring. Let $U$ be any multiplicative system. Let $\tau$ be the test ideal for $R$ and $\tau_{U}$ be the test ideal for $U^{-1} R$. Then $\tau U^{-1} R=\tau_{U}$, i.e., the test ideal localizes.

Moreover, let $R$ be a local reduced ring. If for some prime ideal $P$ the ring $R_{P}$ is $\mathbb{Q}$ Gorenstein then $\tau_{P}=\tau R_{P}$.

Proof. To start with we will assume that $U=R-P$ for some $P \in \operatorname{Spec} R$ and $R_{P}$ is $\mathbb{Q}$-Gorenstein.

First we show $\tau R_{P} \subseteq \tau_{P}$. Recall that $\tau_{P}=\cap I_{t}: I_{t}^{*}$ for any sequence of Gorenstein ideals, $I_{t}$, in $R_{P}$ such that $I_{t} \subseteq P^{t}$. If $J$ is any canonical ideal for $R_{P}$, then for any $r \in R_{P}, R_{P}$ is $\mathbb{Q}$-Gorenstein with respect to $r J$, so for any $t$ we may take a canonical ideal, $J_{t}$, contained in $P^{t}$. Take $x_{t, 1} \in J_{t}^{(h)} R_{P}$ such that $\left(J_{t} R_{P}\right)^{(h)}=\left(x_{t, 1}\right) R_{P}$. Suppose the height of $P$ is $k$. Pick $x_{t, 2}, \ldots, x_{t, k} \in P^{t}$ so that $x_{t, 1}, \ldots, x_{t, k}$ are parameters in $R$. The ideals $\left(J_{t}, x_{t, 2}, \ldots, x_{t, k}\right) R_{P}$ form a sequence of Gorenstein ideals so that in particular it suffices to show (we drop the subscript $t$ for the rest of the argument) that $\tau\left(\left(J, x_{2}, \ldots, x_{k}\right) R_{P}\right)^{*} \subseteq\left(J, x_{2}, \ldots, x_{k}\right) R_{P}$.

Take $u \in\left(J, x_{2}, \ldots, x_{k}\right) R_{p}^{*}$. Then for each $q$ there is an $f_{q} \in R-P$ with $c u^{q} f_{q} \in$ $\left(J, x_{2}, \ldots, x_{k}\right)^{(q)} R$. Let $P_{1}, \ldots, P_{m}$ be the associated primes of $\left(x_{1}, \ldots, x_{k}\right)$ with $P=P_{1}$ and let $\mathfrak{p}_{1}, \ldots, \mathfrak{p}_{m}$ be the primary components. Set $S=\left(R-\cup P_{i}\right)^{-1} R$. Since $\left(x_{1}, \ldots, x_{k}\right)^{[q]} S$ has no embedded components, its primary components are $p_{1}^{[q]} S, \ldots, p_{m}^{[q]} S$. Note that since $\left(J R_{P}\right)^{(h)}=x_{1} R_{P}$, we have for all $q$ there exists $g_{q} \in R-P$ that $g_{q} J^{(q)} \subseteq g_{q} J^{(q)} \subseteq\left(x_{1}^{\lfloor q\rfloor}\right)$ for all $q$. Now $c u^{q} f_{q} g_{q} \in\left(g_{q} J^{[q]}, x_{2}^{q}, \ldots, x_{k}^{q}\right) R \subseteq\left(x_{1}^{[q]}, x_{2}^{q}, \ldots, x_{k}^{q}\right) R$, and hence $c u^{q} x_{1}^{q-\left\lfloor h_{h}^{q}\right\rfloor} f_{q} g_{q} \in$ $\left(x_{1}^{q}, \ldots, x_{k}^{q}\right) R$. It follows that $c u^{q} x_{1}^{\left.q-1 q_{h}\right]} \in\left(x_{1}, \ldots, x_{k}\right) S^{[q]}: f_{q} g_{q} \subseteq \mathfrak{p}_{1}^{[q]} S$ since $f_{q} g_{q} \notin P_{1}$. For some $b_{i}, P_{i}^{b_{i}} \subseteq \mathfrak{p}_{i} S$. Take $f \in P_{2}^{b_{2}} \ldots P_{m}^{b_{m}}-P_{1}$. Then $c u^{q} x_{1}^{q-l_{k}^{q]}} f^{q} \in\left(x_{1}, \ldots, x_{k}\right) S^{[q]}$. Clearing denominators, $c u^{q} x_{1}^{q-\left\lfloor h_{h}\right\rfloor} f^{q} h_{q} \in\left(x_{1}^{q}, \ldots, x_{k}^{q}\right) R$ for some $h_{q} \notin \cup P_{i}$. Here $x_{1}, \ldots, x_{k}, h_{q}$ is a regular sequence and so the $h_{q}$ cancels. The $x_{1}$ powers also cancel to give $c u^{q} f^{q} \in\left(x_{1}^{|q|}, x_{2}^{q}, \ldots, x_{k}^{q}\right) R$. Let $J=(a: b)$ as in Remark 2.1. Then $\left(a^{n} c\right) u^{q} f^{q} b^{q} \in$ $\left(a^{q}, b^{q} x_{2}^{q}, \ldots, b^{q} x_{k}^{q}\right)$. In particular $u f b \in\left(a, b x_{2}, \ldots, b x_{k}\right)^{*}$ and so $\tau u f b \subseteq\left(a, b x_{2}, \ldots, b x_{k}\right)$. 
Thus $\tau(u f) \subseteq\left((a: b), x_{2}, \ldots, x_{k}\right)=\left(J, x_{2}, \ldots, x_{k}\right)$, and hence $\tau R_{P} \subseteq \tau_{P}$.

We note that this argument does not depend on $R$ being local.

By Lemma 3.2 it now suffices to show that $\operatorname{Ann}_{E(R / P)} \tau R_{P} \subseteq 0_{E(R / P)}^{*}$. Keep $J, x_{1}, \ldots, x_{k}$ as above. As above, take any $\eta=\left[z+\left(x_{1}^{i} J, \ldots, x_{k}^{i}\right) R_{P}\right] \in \mathrm{Ann}_{E(R / P)} \tau R_{P}$. Then $\tau\left(x_{1} \ldots x_{n}\right)^{s} z f \in\left(x_{1}^{s+l} J, \ldots, x_{k}^{s+l}\right)$ for some $f \in R-P$ and $s \in \mathbb{N}$. Extend $x_{1}, \ldots, x_{k}$ to $x_{1}, \ldots, x_{n}$ a full system of parameters for $R$. Then for all $H \in \mathbb{N}, \tau\left(x_{1} \cdots x_{n}\right)^{s} z f \in$ $\left(x_{1}^{s+\imath} J, \ldots, x_{k}^{s+t}, x_{k+1}^{H}, \ldots, x_{n}^{H}\right)$. Thus $v=\left[z f+\left(x_{1}^{\imath} J, \ldots, x_{k}^{t}, x_{k+1}^{H}, \ldots, x_{n}^{H}\right)\right] \in \mathrm{Ann}_{E} \tau=0_{E}^{*}$. This gives $v^{[q]}=\left[(z f)^{q}+\left(x_{1}^{q t} J^{[q]}, \ldots, x_{k}^{q t}, x_{k+1}^{q H}, \ldots, x_{n}^{q H}\right)\right] \in 0_{F(E)}^{*}$. Now for some $s$ (which may depend on $H$ ) we have

$$
\tau\left(x_{1}^{q t s} \cdots x_{k}^{q t s} x_{k+1}^{q H s} \cdots x_{n}^{q H s}\right)(z f)^{q} \in\left(x_{1}^{q t(s+1)} J^{[q]}, \ldots, x_{k}^{q t(s+1)}, x_{k+1}^{q H(s+1)}, \ldots, x_{n}^{q H(s+1)}\right) .
$$

Multiply by $g_{q} \in R-P$ where $g_{q} J^{(q)} \subseteq x_{1}^{\left\lfloor\frac{q}{b}\right\rfloor}$ to get a monomial ideal and so cancel $x$ terms to get

$$
\tau(z f)^{q} g_{q} \in\left(x_{1}^{q t+\mid q)}, \ldots, x_{k}^{q t}, x_{k+1}^{q H}, \ldots, x_{n}^{q H}\right)
$$

Since this is true for any $H$, we have $\tau(z f)^{q} g_{q} \in\left(x_{1}^{q t+\left(q_{h}^{q}\right\rfloor}, \ldots, x_{k}^{q t}\right)$ or $\tau z^{q} \in$ $\left(x_{1}^{\left.q t+1 q^{q}\right\rfloor}, \ldots, x_{k}^{q t}\right) R_{P}$. Multiplying both sides by $x_{1}$ we see that $x_{1} \tau z^{q} \in\left(x_{1}^{q t} J^{(q)}, \ldots, x_{k}^{q t}\right) R_{P}$. In particular $x_{1} \tau \eta^{[q]}=0 \in F^{e}(E(R / P))$ for all $q$ and thus $\eta \in 0_{E(R / P)}^{*}$.

Now let $R$ be $\mathbb{Q}$-Gorenstein and let $U$ be any multiplicative system. Let $I \subseteq U^{-1} R$ and $z \in\left(I U^{-1} R\right)^{*}$. For any $P \in \operatorname{Spec} U^{-1} R, z / 1 \in\left(I R_{P}\right)^{*}$, hence $\tau(z / 1) \subseteq I R_{P}$. Thus $\tau(z / 1) \subseteq I U^{-1} R$, and hence $\tau U^{-1} R \subseteq \tau_{U}$. If $w \in \tau_{U}$ then our observation that the first part of the proof holds for any ring shows that $w / 1 \in \tau_{P}=\tau R_{P}$ for all $P \in \operatorname{Spec} U^{-1} R$. Hence $w \in \tau U^{-1} R$.

Corollary 3.4. If $R$ is a weakly F-regular $\mathbb{Q}$-Gorenstein reduced ring then $R$ is F-regular.

Proof. By the proof of Lemma 3.2, $\tau R_{P} \subseteq \tau_{P}$ for a non-local $\mathbb{Q}$-Gorenstein reduced ring. If $R$ is weakly $F$-regular then $1 \in \tau$ and thus $1 \in \tau_{P}$ and hence $R_{P}$ is weakly F-regular.

Corollary 3.5. Let $R$ be an excellent weakly F-regular ring. Then $R_{p}$ is F-regular and $\mathbb{Q}$-Gorenstein for any prime ideal $P$ of height two.

Proof. $\quad R$ weakly F-regular implies $R$ is F-rational hence pseudorational [9] and so $\mathbb{Q}$-Gorenstein at height two primes [5]. Hence by Theorem 3.3, for any prime, $P$, of height at most two, $1 \in \tau R_{P}=\tau_{P}$ so that $R_{P}$ is weakly F-regular.

Corollary 3.6. If $R$ is a reduced excellent local Cohen-Macaulay $\mathbb{Q}$-Gorenstein ring then the defining ideal of the non-F-regular locus is $\operatorname{rad}(\tau)$ and $\tau$ is in fact the completely stable test ideal. 
Proof. $R_{P}$ is weakly F-regular if and only if $1 \in \tau_{p}=\tau R_{p}$. Thus $R_{p}$ is weakly Fregular if and only if $P \notin V(\tau)$. Moreover, $R$ has a completely stable test element by [4, 7.32] (or 4.2) and hence by $[3,8.23] \tau\left(R_{P}\right)=\tau\left(\widehat{R_{P}}\right) \cap R_{P}$. Thus any $c \in \tau$, by Theorem 3.3 , is in $\tau\left(\widehat{R_{P}}\right)$ and so is also a completely stable test element.

Notice a consequence of this last corollary is that, for reduced excellent local $\mathbb{Q}$-Gorenstein rings, if $R_{c}$ is F-regular then $c$ has a power which is a completely stable test element. This is a weaker version of the theorem on existence of test elements, Theorem 4.2.

\section{Existence of test elements}

The basis of most existence results for test elements is that if $R$ is F-finite and $c$ is such that $R_{c}$ is strongly F-regular, then $c$ has a power in the test ideal [2, 3.4]. Difficulties arise if the ring is not F-finite. The idea of the gamma construction is when given an excellent local ring $S$ which is not $F$-finite to create a flat, purely inseparable extension ring $R^{\Gamma}$ of $R=\hat{S}$ such that $\left(R^{\Gamma}\right)^{1 / p}$ is module finite over $R^{\Gamma}$. This allows one to pass from the ring to a gamma construction, use F-finiteness, and then descend back to $R$ and then $S$. This technique is developed in [4]. There one assumes the original ring $S$ (or $S$ localized at some element) is weakly F-regular and one needs to deduce that the gamma construction is strongly $\mathrm{F}$-regular. A key point is that weakly F-regular Ffinite Gorenstein rings are strongly $F$-regular. We seek to improve this existence result by noting that in fact weak F-regularity is strong F-regularity for $\mathbb{Q}$-Gorenstein rings [6]. We refer to [4] for the details and proofs involving $R^{\Gamma}$, but we list the basic definitions below.

Let $(R, m, K)$ be a complete ring. Let $\Gamma$ be a cofinite subset of a p-base $\Lambda$. Define $K_{e}^{\Gamma}=K\left[\lambda^{1 / p^{e}}: \lambda \in \Gamma\right]$. Set $R^{\Gamma}=\bigcup_{e} K_{e}^{\Gamma}[[R]]$ (where for a field $L \supseteq K, L[[R]]$ is the completion of $L \otimes_{K} R$ at $m\left(L \otimes_{K} R\right)$ ). If $R$ is a finitely generated algebra over a complete local ring $B$, then $R^{\Gamma}=R \otimes_{B} B^{\Gamma}$. Then $R^{\Gamma}$ is F-finite as well as faithfully flat and purely inseparable over $R$, with Gorenstein fibers.

Hence if $J$ is a canonical ideal of a Cohen-Macaulay ring, $R$, then $J R^{\Gamma}$ is a canonical ideal for $R^{\Gamma}[1,3.3 .14]$. Also the primary decompositions are preserved by flatness, i.e., $J^{(n)} R^{\Gamma}=\left(J R^{\Gamma}\right)^{(n)}$, so if $R$ is $\mathbb{Q}$-Gorenstein then $R^{\Gamma}$ is also $\mathbb{Q}$-Gorenstein. Similarly, for $U$ any multiplicative system of $R$, if $U^{-1} R$ is $\mathbb{Q}$-Gorenstein so is $U^{-1} R^{\Gamma}$.

Now the existence of test elements follows directly from [4]. First we have an analogue of $[4,6.19]$.

Lemma 4.1. Let $R$ be a reduced algebra of finite type over a complete local ring $B$. Let $c$ be such that $R_{c}$ is weakly F-regular and $\mathbb{Q}$-Gorenstein. Then there exists a choice for $\Gamma$ such that $R_{c}^{\Gamma}$ is weakly F-regular and $\mathbb{Q}$-Gorenstein.

Proof. The proof is identical to $[4,6.19]$ by observing that one does not actually require that $R_{c}$ is Gorenstein, only that the weakly F-regular locus of $R_{c}^{\Gamma}$ is the same as the strongly F-regular locus and this follows since $R_{c}^{\Gamma}$ is $\mathbb{Q}$-Gorenstein. 
The following theorem is a strengthening of [4, 7.32]. As in that result, we require that if $R_{c}$ is strongly F-regular then $c$ has a power which is a completely stable element and again $[4,5.10]$ is given as a reference even though it does not precisely state this (it is an immediate consequence of that theorem).

Theorem 4.2. Let $R$ be a reduced algebra of finite type over a reduced excellent local ring $B$. Let $c$ not in any minimal prime be such that $R_{c}$ is weakly $F$-regular and $\mathbb{Q}$ Gorenstein. Then $c$ has a power which is a completely stable test element.

Proof. Since the fibers of $B \rightarrow \hat{B}$ are smooth we have that $R \rightarrow R \otimes_{B} \hat{B}$ is regular thus $\left(R \otimes_{B} \hat{B}\right)_{c}$ is weakly F-regular by Theorem 3.3 and $[4,7.25(\mathrm{a})]$.

Apply Lemma 4.1 to get a choice for $\Gamma$ such that $\left(R \otimes_{B} \hat{B}\right)_{c}^{\Gamma}$ is strongly F-regular. Hence $c$ has a power which is a completely stable test element in $\left(R \otimes_{B} \hat{B}\right)^{\Gamma}[4,5.10]$. Since $\left(R \otimes_{B} \hat{B}\right)^{\Gamma}$ is faithfully flat over $R$, this power is also a completely stable test element for $R[4,6.14(\mathrm{c})]$.

Theorem 4.3. Let $R$ be of finite type over an excellent local ring $B$. The $\mathbb{Q}$-Gorenstein, weakly F-regular locus is open.

Proof. Say $R_{p}$ is weakly F-regular with canonical ideal $J$. Let $S=R \otimes_{B} \hat{B}$. Then $S$ is $\mathbb{Q}$-Gorenstein and for any prime ideal $Q$ of $S$ lying over $P$ the map $R_{P} \rightarrow S_{Q}$ is faithfully flat. The fibers are regular, so $Q S_{Q}=\left(P, w_{1}, \ldots, w_{l}\right) S_{Q}$. Also, $S_{Q}$ is $\mathbb{Q}$ Gorenstein. Let $u$ be the socle element of $R_{P} /\left(x^{t} J, y_{2}, \ldots, y_{k}\right)$. Then the image of $u$ is the socle element in $S_{Q} /\left(x^{t} J, y_{2}, \ldots, y_{k}, w_{1}, \ldots, w_{l}\right)$. Thus if $\left(x^{t} J, y_{2}, \ldots, y_{k}, w_{1}, \ldots, w_{l}\right)$ fails to be tightly closed in $S_{Q}$ we get the image of $u$ in $\left(\left(x^{t} J, y_{2}, \ldots, y_{k}, w_{1}, \ldots, w_{l}\right) S_{Q}\right)^{*}$, and hence $u \in\left(\left(x^{t} J, y_{2}, \ldots, y_{k}\right) R_{P}\right)^{*}$ since $R$ and $S$ have a common completely stable test element. Then for $\Gamma$ small enough, $S_{P}^{r}$ is strongly F-regular. The strongly F-regular locus of $S^{\Gamma}$ is open so there exists $c \in R-P$ such that $S_{c}^{\Gamma}$ is strongly F-regular, and hence $R_{c}$ is F-regular. It is easy to see that a further localization at $c^{\prime}$ makes $R_{c c^{\prime}}$ both $\mathbb{Q}$-Gorenstein and (weakly) F-regular.

Corollary 4.4. The completely stable test ideal in a local excellent weakly F-regular domain has height at least three.

Proof. Let $\tau^{\prime}$ be the completely stable test ideal for $R$. If we localize at any height two prime, $P$, the ring is F-regular and $\mathbb{Q}$-Gorenstein by Corollary 3.5. Since we can preserve $\left(J R_{P}\right)^{(n)}=\left(x_{1}\right) R_{P}$ by localizing at a single element, we may take $c \in R-P$ such that $R_{c}$ is $\mathbb{Q}$-Gorenstein. By Theorem 4.3 , the non-F-regular locus of $R_{\mathrm{c}}$ is closed, since $R_{c}$ is an algebra of finite type over $R$. If $I$ is the defining ideal, then since $I$ has height at least three we may take $d \in I-P$. Hence $R_{c d}$ is $\mathbb{Q}$-Gorenstein and F-regular. By Theorem 3.2, $c d$ has some power in the completely stable test ideal, but $c d \notin P$ and hence $\tau^{\prime} \nsubseteq P$. 


\section{REFERENCES}

1. W. Bruns and J. Herzog, Cohen-Macaulay rings (Cambridge University Press, 1993).

2. M. HOCHSTER AND C. HUNEKE, Tight closure and strong F-regularity, Soc. Math. France Memoire 38 (1989), 119-133.

3. M. Hochster and C. Huneke, Tight closure, invariant theory, and the Briançon-Skoda theorem, J. Amer. Math. Soc. 3 (1990), 31-116.

4. M. Hochster AND C. HUNEKE, F-regularity, test elements, and smooth base change, Trans. Amer. Math. Soc. 346 (1994), 1-62.

5. J. Lipman, Rational singularities with applications to algebraic surfaces and unique factorization, I.H.E.S. Publ. Math. 36 (1969), 195-279.

6. B. MaCCRimmon, Weak F-regularity is strong F-regularity for rings with isolated non-QGorenstein points, Trans. Amer. Math. Soc., to appear.

7. K. E. SMITH, Tight closure of parameter ideals, Invent. Math. 115 (1994), 41-60.

8. K. E. Smith, Test ideals in local rings, Trans. Amer. Math. Soc. 347 (1995), 3453-3472.

9. K. E. Sмiтh, F-rational rings have rational singularities, Amer. J. Math. 119 (1997), 159-180.

Department of Mathematics

UNIVERSITY OF MISSOURI

Columbia, MO 65211

U.S.A.

E-mail address: aberbach@math.missouri.edu 